\title{
MLL-MLLT10 fusion in acute monoblastic leukemia: variant complex rearrangements and 11q proximal breakpoint heterogeneity
}

\author{
Cristina Morerio ${ }^{a}$, Annamaria Rapella ${ }^{a}$, Cristina Rosanda ${ }^{a}$, Edoardo Lanino ${ }^{a}$, Luca Lo Nigro ${ }^{b}$, \\ Andrea Di Cataldo ${ }^{\mathrm{b}}$, Emanuela Maserati ${ }^{\mathrm{c}}$, Francesco Pasquali ${ }^{\mathrm{c}}$, Claudio Panarello $^{\mathrm{a}, *}$ \\ ${ }^{a}$ Dipartimento di Ematologia ed Oncologia Pediatrica, Istituto Giannina Gaslini, L.go G. Gaslini 5, 16148 Genova, Italy \\ ${ }^{\mathrm{b}}$ Centro di Ematologia ed Oncologia Pediatrica, Azienda Policlinico, Università di Catania, Catania, Italy \\ ${ }^{\mathrm{c}}$ Sezione di Biologia e Genetica, Dipartimento di Scienze Biomediche Sperimentali e Cliniche, Università dell'Insubria, Varese, Italy \\ Received 25 September 2003; received in revised form 24 November 2003; accepted 25 November 2003
}

\begin{abstract}
Cytogenetic studies of acute monoblastic leukemia cases presenting MLL-MLLT10 (alias MLL$A F 10$ ) fusion show a broad heterogeneity of chromosomal breakpoints. We present two new pediatric cases (French-American-British type M5) with MLL-MLLT10 fusion, which we studied with fluorescence in situ hybridization. In both we detected a paracentric inversion of the 11q region that translocated onto chromosome 10p12; one case displayed a variant complex pattern. We review the cytogenetic molecular data concerning the proximal inversion breakpoint of $11 \mathrm{q}$ and confirm its heterogeneity. (C) 2004 Elsevier Inc. All rights reserved.
\end{abstract}

\section{Introduction}

Chromosome rearrangements involving the mixed lineage leukemia $(M L L)$ gene at the 11q23 locus occur in $~ 5-10 \%$ of acute leukemias, mainly of myeloid or poorly differentiated lymphoid origin, and are associated with poor prognosis [1-3]. To date, 37 partners of $M L L$ have been identified [4]. In particular, two genes may be involved in $\mathrm{t}(10 ; 11)$ : SSH3BP1 (alias $A B I-1$; also $A B I 1$ ), which is a human homolog to the mouse Abl interactor 1 at 10p11.2, and which has been described in only three cases of pediatric acute monocytic leukemia [5-7], and MLLT10 (alias AF10) at 10p13, which is more commonly fused to $M L L$ [8]. The majority of the other $M L L$ rearrangements result from a reciprocal translocation, but MLL-MLLT10 fusions require more complex chromosome rearrangements. These various mechanisms have recently been elucidated by Van Limbergen et al. [3]. All the $11 \mathrm{q} 23$ translocations involving $M L L$ lead to fusion genes in which the $5^{\prime}$ part belongs to $M L L$ [9]. The expression of a chimeric product requires the same orientation of the two fused genes: because the direction of $M L L$ transcription is centromere to telomere, an inversion has to occur if the partner gene is oriented in the opposite way [9]. Four recom-

\footnotetext{
* Corresponding author. Tel.: +39-010-5636656; fax: +39-010-
} 3761017.

E-mail address: claudiopanarello@ospedale-gaslini.ge.it (C. Panarello). bination patterns have been proposed: types 1 and 2 result from inversion of 11q13 q14-q23 involving the $M L L$ locus, followed by translocation with (type 1) or insertion into (type 2) 10p12; the two other types require the inversion of MLLT10 gene followed by translocation with (type 3) or insertion into (type 4) 11q23 [3]. We present two new cases of pediatric acute monoblastic leukemia with MLL-MLLT10 fusion of type 2 (according to Van Limbergen et al. [3]), one of them showing involvement of a third chromosome. We also briefly discuss the variability of the breakpoint on 11q centromeric to $M L L$ gene in this particular mechanism.

\section{Case reports}

\subsection{Patient 1}

At 4 months of age, hyperleukocytosis (a white blood cell [WBC] count of $41 \times 10^{9} / \mathrm{L}$ ) prompted a diagnosis of acute myeloid leukemia (AML), French-American-British (FAB) type M5a. Blast immunophenotype was characterized by a positivity of HLA-DR, CD45, CD34, CD33, CD11a, CD11b, CD11c, CD15, CD7, CD19, and CD38. The child was enrolled in protocol AML-BFM 93 [10] and achieved clinical remission. At 4 months from diagnosis, however, leukemia recurred, with the presence of blasts in cerebrospinal fluid and partial (9\%) marrow involvement. Bone marrow transplantation (BMT) from a partially matched related donor was performed after conditioning 
with busulfan-cyclophosphamide-melphalan and intrathecal cytarabine. At 5 months after BMT, a second hematological and central nervous system (CNS) relapse occurred; further remission with full donor chimerism was achieved following enrollment in IDA FLAG/FLAG protocols [11], as confirmed by the absence of recipient-derived DNA through short tandem repeat polymorphism analysis of BM cells. A second BMT from the same donor was successfully performed, and at writing the patient was alive and in third complete remission, 24 months after retransplant.

\subsection{Patient 2}

When the patient was 8 months old, a diagnosis was made of AML FAB M5 with CNS and cutaneous involvement. The patient was enrolled in an ongoing international protocol for infant leukemia (Interfant 99: dexamethasone, vincristine, daunorubicin, cytarabine, L-asparaginase, methotrexate, cyclophosphamide, and mercaptopurine) and achieved hematological, liquoral, and cutaneous remission. After 8 months of treatment, hematological and CNS relapse occurred. A second-line chemotherapy course (FLAD: fludarabine, cytarabine, and liposomal daunorubicin), delayed due to interstitial pneumonia, produced a partial response with persistence of residual blasts in bone marrow, CNS and cutis (leukemoids). The child underwent allogeneic BMT from a match-unrelated donor (female) after conditioning with busulfan-thiotepa-etoposide and intrathecal cytarabine; however, lymph node relapse occurred 2 months after transplant and the child died shortly after with progressive disease.

\section{Materials and methods}

At onset or relapse (or both), chromosome analyses were performed on unstimulated peripheral blood (PB) and bone marrow (BM) from both patients, and on lymph node blasts from patient 2, using a QFQ-banding technique. A minimum of 20 metaphases were screened. Karyotype designation was according to International System for Human Cytogenetic Nomenclature (ISCN 1995) [12]. Fluorescence in situ hybridization (FISH) analyses were performed according to the manufacturer's protocols using whole-chromosome 7, 10, and 11 painting probes (wcp) (Appligene Oncor-Qbiogene, Illkirch, France) and using the LSI MLL dual-color probe

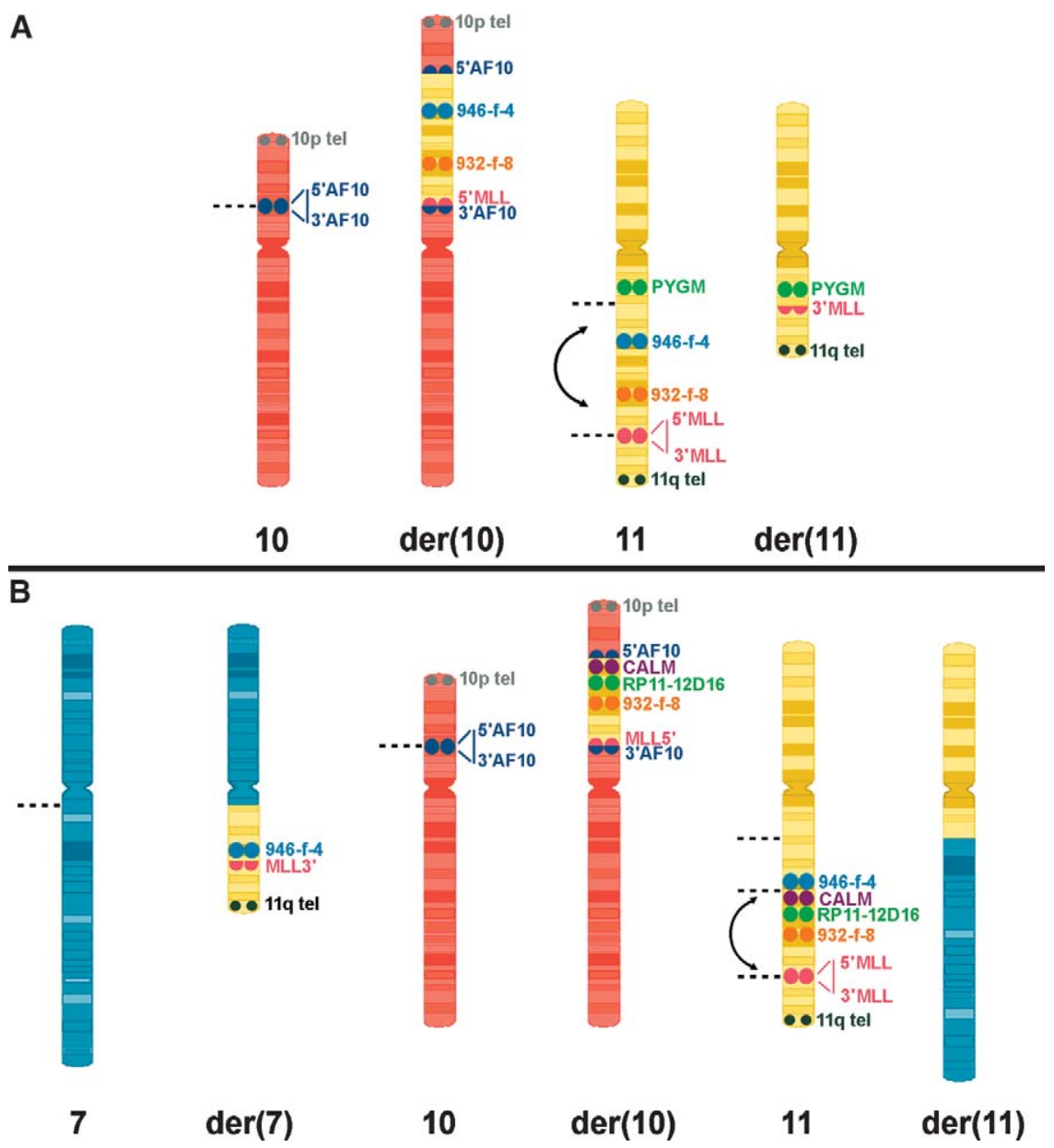

Fig. 1. Ideograms of the 11q arm, with schematic representation of FISH studies in $(A)$ patient 1 and $(B)$ patient 2 . Dotted lines show breakpoints; arrowheads indicate the regions of $11 \mathrm{q}$ chromosome inversion. 
(Vysis, Downers Grove, IL), which recognizes the $M L L$ gene at 11q23. Human DNA inserts of yeast artificial chromosome (YAC) were amplified using Alu-polymerase chain reaction as previously described [13]. YAC or bacterial artificial chromosome (BAC) probes were labeled with nick translation with biotin-16- or digoxigenin-11-dUTP (Roche, Mannheim, Germany) and visualized using streptavidin-Cy3 (Amersham Pharmacia Biotech, Buckinghamshire, UK) and sheep antidigoxigenin-FITC (Roche). The following YAC or BAC probes were used: RP11-418C1 located telomeric to MLLT10 and also containing the $5^{\prime}$ part of the gene cohybridized with RP11-249M6 located centromeric to MLLT10 and also containing the $3^{\prime}$ part of the gene; RP11141J21 cohybridized with RP11-665N17, both located at 11q13.1, centromeric and telomeric to the marker PYGM (muscle glycogen phosphorylase gene), respectively [9]; YAC 946_f_4 (11q14.1) cohybridized with YAC 932_f_8 (11q21); RP11-103I15 containing the PICALM gene (alias CALM,$C L T H)$ at 11q14.2 cohybridized with RP11-12D16 located telomeric to PICALM; subtelomeric 10p RP11-145I2 cohybridized with subtelomeric 11q RP11-209L12. Images were captured on a Zeiss Axioplan microscope (Carl Zeiss, Jena, Germany) equipped with a charge-coupled device (CCD) camera. Adobe Photoshop software (Adobe Systems, Seattle, WA) was used to pseudocolor and merge images.

\section{Results and discussion}

\subsection{Patient 1}

Cytogenetic analysis performed on BM blasts revealed a chromosome 10;11 rearrangement. FISH studies are outlined in Fig. 1A. FISH analyses with wcp 10 and 11 and with 10p and $11 \mathrm{q}$ subtelomeric probes revealed insertion of chromosome $11 \mathrm{q}$ material into the short arm of chromosome 10 . Hybridization signals for the MLL dual probe were split, with localization of the $5^{\prime}$ part of $M L L$ on the proximal region of $11 \mathrm{q}$ inserted into $10 \mathrm{p}$, while the $3^{\prime}$ part of $M L L$ was retained on the derivative chromosome 11. Dual-color FISH showed that probes for the $3^{\prime}$ (RP11-249M6) and $5^{\prime}$
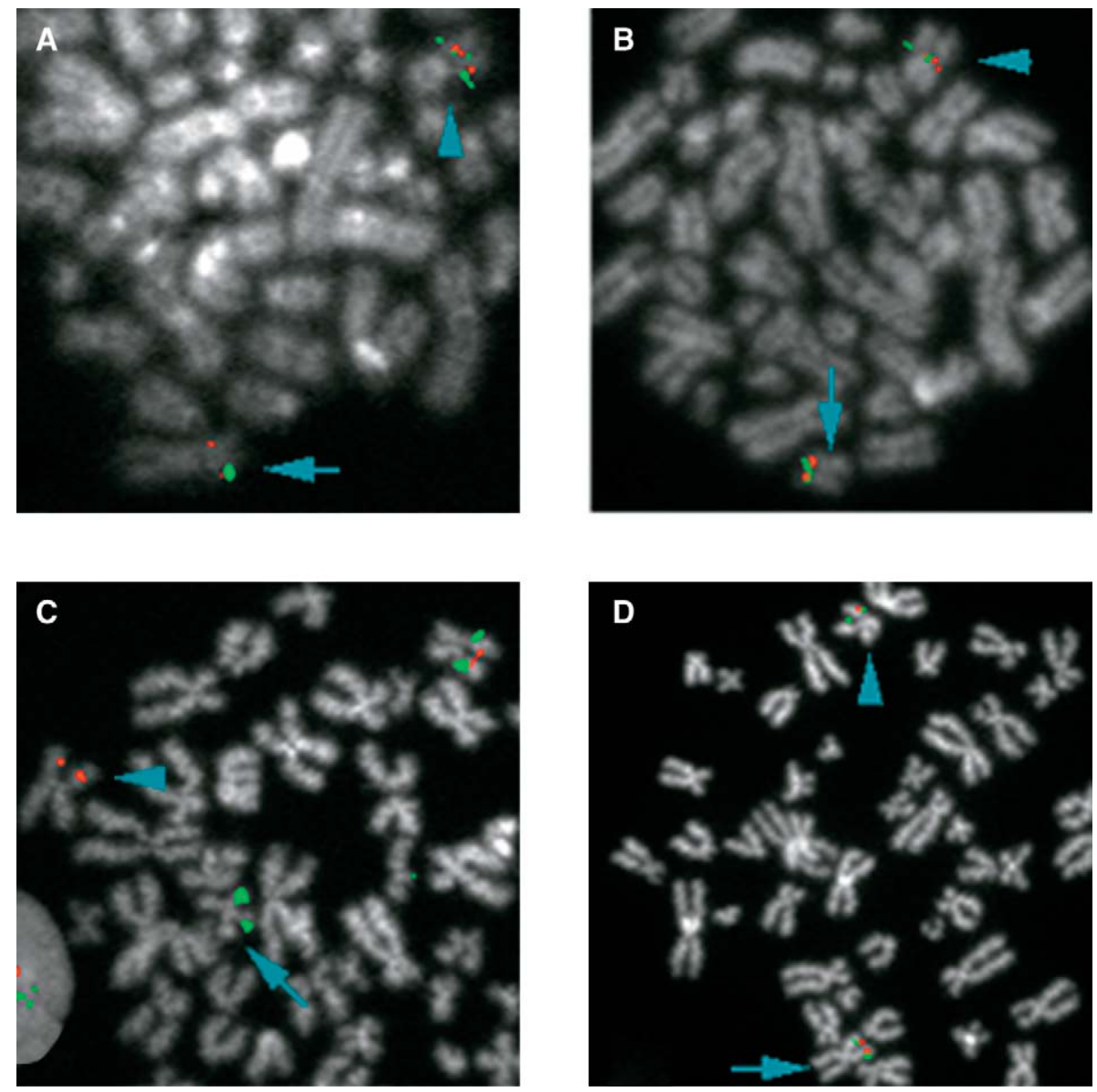

Fig. 2. FISH analyses of the 11q proximal inversion breakpoint in metaphase cells of $(A, B)$ patient 1 and $(C, D)$ patient 2. (A) YACs 946_f_4 at 11q14.1 (green) and 932_f_8 at 11q21 (red) were present on the normal chromosome 11 (arrowhead) and on the der(10) (arrow). (B) RP11-141J21 (red) and RP11-665N17 (green) at 11q13.1, centromeric and telomeric to the PYGM gene, respectively, both localized on the normal chromosome 11 (arrowhead) and on the der(11) (arrow). (C) Hybridization signals of YACs 946_f_4 at 11q14.1 (green) and 932_f_8 at 11q21 (red) are localized on the der(7) (arrow) and the der(10) (arrowhead), respectively. (D) RP11-103I15 (red) containing the PICALM gene at 11q14.2 and RP11-12D16 (green) telomeric to PICALM were present on the normal chromosome 11 (arrowhead) and on the der(10) (arrow). 
Table 1

Proximal inversion breakpoints at $11 \mathrm{q}$ detected with FISH

\begin{tabular}{|c|c|c|c|c|}
\hline Case $^{\mathrm{a}}$ & Mechanism type ${ }^{b}$ & Probe & Breakpoint region & Reference \\
\hline $\mathrm{a}$ & 1 variant & PYGM/3.16 & $11 \mathrm{q} 10 \sim 11 \mathrm{q} 13.1$ & Patient 3 in Beverloo et al., 1995 [9] \\
\hline $\mathrm{b}$ & 1 & PYGM/3.16 & $11 \mathrm{q} 13.1 \sim 11 \mathrm{q} 21$ & Patient 5 in Beverloo et al., 1995 [9] \\
\hline $\mathrm{c}$ & 2 variant & 946_f_4/932_f_8 & $11 \mathrm{q} 14.1 \sim 11 \mathrm{q} 21$ & Patient 1 in Van Limbergen et al., 2002 [3] \\
\hline $\mathrm{d}$ & 1 & RP11-665N17/946_f_4 & $11 \mathrm{q} 13.1 \sim 11 \mathrm{q} 14.1$ & Patient 1 , present study \\
\hline $\mathrm{e}$ & 2 variant & 946_f_4/RP11-103I15 & $11 \mathrm{q} 14.1 \sim 11 \mathrm{q} 14.2$ & Patient 2 , present study \\
\hline
\end{tabular}

a Cases correspond to a-e in Fig. 3.

b Classification according to Van Limbergen et al. [3].

(RP11-418C1) regions of MLLT10, which normally hybridize on the short arm of chromosome 10, were located at either side of the inserted 11q segment. Hybridization signals for YAC 946_f_4 (11q14.1) and YAC 932_f_8 (11q21) both localized on the inserted 11q segment (Fig. 2A), whereas RP11-141J21 and RP11-665N17 (PYGM) signals were retained on the derivative chromosome 11 (Fig. 2B). The karyotype thus was 46,XY,ins(10;11)(p12;q23q13).

\subsection{Patient 2}

The karyotype of $\mathrm{PB}$ and $\mathrm{BM}$ blasts at onset and at relapse was described as $46, \mathrm{XY}, \mathrm{t}(7 ; 11 ; 10)(\mathrm{q} 21 ; \mathrm{q} 21 ; \mathrm{p} 15)$. At second relapse after BMT, the karyotype of lymph node blasts revealed an additional chromosomal anomaly: 46,XY,t(1;4)(p32;q31),t(7;11;10)(q21;q21;p15). FISH analyses summarized in Fig. 1B detail the complex rearrangement that occurred. FISH with wcp 7, 10, and 11 and with subtelomeric probes for 10p-tel (RP11-145I2) and 11qtel (RP11-209L12) revealed an insertion of chromosome 11 material into the short arm of chromosome 10 and the reciprocal translocation between the telomeric portion of 11q and part of chromosome 7 long arm. Hybridization signals for the MLL dual probe were split: the $5^{\prime}$ part of $M L L$ was inserted into $10 \mathrm{p}$ and the $3^{\prime}$ part of $M L L$ was translocated onto the derivative chromosome 7; no $M L L$ signal was observed on the derivative chromosome 11. Dualcolor FISH using probes for the 3' (RP11-249M6) and 5' (RP11-418C1) parts of MLLT10 localized both signals on the short arm of the derivative chromosome 10, split at the proximal and distal ends of 11q inserted segments, respectively. Hybridization signals for YAC 946_f_4 (11q14.1) and YAC 932_f_8 (11q21) were localized on derivative chromosome 7 and on the distal part of inserted 11q segment, respectively (Fig. 2C). Cohybridization between PICALM probes RP11-103I15 and RP11-12D16 showed both signals retained on the distal part of the $11 \mathrm{q}$ inserted segment (Fig. 2D). After FISH analysis, the revised karyotype was $46, \mathrm{XY}, \mathrm{t}(7 ; 10 ; 11)(7 \mathrm{pter} \rightarrow 7 \mathrm{q} 11.2:: 11 \mathrm{q} 13 \rightarrow 11 \mathrm{q} 14.1:: 11 \mathrm{q} 23 \rightarrow$ 11qter;10pter $\rightarrow 10$ p12::11q14.2 $\rightarrow 11 \mathrm{q} 23:: 10 \mathrm{p} 12 \rightarrow 10 \mathrm{qter} ; 11$ $\mathrm{pter} \rightarrow 11 \mathrm{q} 13:: 7 \mathrm{q} 11.2 \rightarrow 7 \mathrm{qter})$.
The $\mathrm{t}(10 ; 11)(\mathrm{p} 12 ; \mathrm{q} 23)$ is associated mainly with acute monocytic leukemias, frequently with CNS disease and/or skin involvement [14], as in the two patients described here. The breakpoint cluster region on 11q23 spans an $8.3-\mathrm{kb}$ segment of genomic DNA, comprising exons 5-10 of the $M L L$ gene [8]. The $\mathrm{t}(10 ; 11)$ produces a MLL-MLLT10 transcript originating from the fusion of the $5^{\prime}$ end of $M L L$ and the $3^{\prime}$ end of MLLT10: the MLL-AF10 chimeric protein contains the AF10 leucine-zipper motif [8] required for immortalization of myeloid progenitors in vitro and endowed with leukemogenic capacity in vivo [15]. Up to now, the pattern of 10;11 chromosomal rearrangements has been determined with molecular cytogenetics in 30 cases $[3,16,17]$. Our two cases show a type 2 pattern according to Van Limbergen's classification: the first case is a more typical ins $(10 ; 11)$ and the second case involves a third chromosome, requiring at least five breakpoints. A variant complex rearrangement involving more than two chromosomes, as in our patient 2 , had been reported in only five previous cases $[3,16,17]$. The mechanism of type 1 and 2 rearrangements requires two breaks (at 11q13 q21 and 11q23, respectively), followed by paracentric inversion of the $11 \mathrm{q}$ segment including the $5^{\prime}$ part of $M L L$ and its insertion or translocation into 10p12. The proximal breakpoint of the

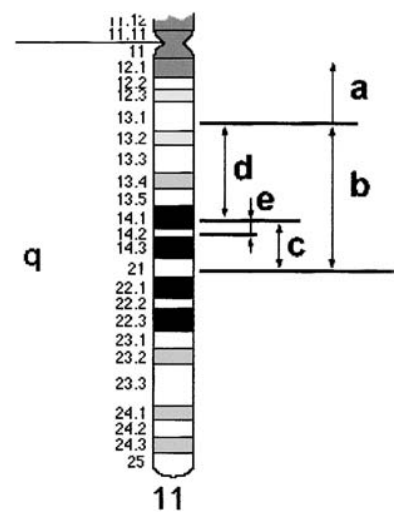

Fig. 3. Schematic illustration of proximal inversion breakpoints: $a$ and $b$ correspond, respectively, to patients 3 and 5 reported by Beverloo et al. [9], $c$ corresponds to patient 1 reported by Van Limbergen et al. [3], $d$ and $e$ to our patients 1 and 2 . 
inverted segment comprising the $M L L$ gene had been identified through molecular cytogenetics in only three cases [3,9] prior to the present two cases (Table 1; Fig. 3). The heterogeneity of the proximal breakpoint locations seems to be confirmed, because the regions defined do not overlap. To better define the complex rearrangements occurring between 10p and 11q, which are often unclear following standard cytogenetic analysis alone, the use of FISH techniques is needed.

\section{Acknowledgments}

The authors thank Antonella Casalaro for technical assistance; Prof. M. Rocchi (University of Bari, Italy) for RP11-145I2, RP11-418C1, RP11-141J21, RP11-665N17, RP11-209L12 BAC clones; Dr. C. Sala (Yac Screening Centre, Milan, Italy) for 807_b_3, 946_f_4, 932_f_8 YAC clones and RP11-249M6, RP11-103I15, RP11-12D16 BAC clones; the Associazione Bambino Emopatico ed Oncologico of Liguria and Association Internationale des Chevaliers des Orders Dynastique de la Maison Royale de Savoie (Vésenaz, Switzerland) for financial support. This work was supported by the Istituto Giannina Gaslini grant Citogenetica Emato-Oncologica and by the Fondazione Gerolamo Gaslini.

\section{References}

[1] Gore L, Ess J, Bitter MA, McGavran L, Meltesen L, Wei Q, Hunger SP. Protean clinical manifestations in children with leukemias containing MLL-AF10 fusion. Leukemia 2000;14:2070-5.

[2] Abdou SM, Jadayel DM, Min T, Swansbury GJ, Dainton MG, Jafer O, Powles RL, Catovsky D. Incidence of $M L L$ rearrangement in acute myeloid leukemia, and a CALM-AF10 fusion in M4 type acute myeloblastic leukemia. Leuk Lymphoma 2002;43:89-95.

[3] Van Limbergen H, Poppe B, Janssens A, De Bock R, De Paepe A, Noens L, Speleman F. Molecular cytogenetic analysis of 10;11 rearrangements in acute myeloid leukemia. Leukemia 2002;16:344-51.

[4] Marschalek R. MLL (myeloid/lymphoid or mixed lineage leukemia). In: Atlas of Genetics and Cytogenetics in Oncology and Haematology [Internet]. Updated November 2002; accessed October 2003. Available at http://www.infobiogen.fr/services/chromcancer/Genes/MLL. html.

[5] Taki T, Shibuya N, Taniwaki M, Hanada R, Morishita K, Bessho F, Yanagisawa M, Hayashi Y. ABI-1, a human homolog to mouse Ablinteractor 1, fuses the $M L L$ gene in acute myeloid leukemia with $\mathrm{t}(10 ; 11)(\mathrm{p} 11.2 ; \mathrm{q} 23)$. Blood 1998;92:1125-30.
[6] Shibuya N, Taki T, Mugishima H, Chin M, Tsuchida M, Sako M, Kawa K, Ishii E, Miura I, Yanagisawa M, Hayashi Y. t(10;11)-acute leukemias with $M L L-A F 10$ and $M L L-A B I 1$ chimeric transcripts: specific expression patterns of $A B I 1$ gene in leukemia and solid tumor cell lines. Genes Chromosomes Cancer 2001;32:1-10.

[7] Morerio C, Rosanda C, Rapella A, Micalizzi C, Panarello C. Is $\mathrm{t}(10 ; 11)(\mathrm{p} 11.2 ; \mathrm{q} 23)$ involving $M L L$ and $A B I-1$ genes associated with congenital acute monocytic leukemia? Cancer Genet Cytogenet 2002; 139:57-9.

[8] Chaplin T, Ayton P, Bernard OA, Saha V, Della Valle V, Hillion J, Gregorini A, Lillington D, Berger R, Young BD. A novel class of zinc finger/leucine zipper genes identified from the molecular cloning of the $\mathrm{t}(10 ; 11)$ translocation in acute leukemia. Blood 1995;85:1435-41.

[9] Beverloo HB, Le Coniat M, Wijsman J, Lillington DM, Bernard O, de Klein A, van Wering E, Welborn J, Young BD, Hagemeijer A, Berger R. Breakpoint heterogeneity in $\mathrm{t}(10 ; 11)$ translocation in AML-M4/ M5 resulting in fusion of $A F 10$ and $M L L$ is resolved by fluorescent in situ hybridization analysis. Cancer Res 1995;55:4220-4.

[10] Creutzig U, Ritter J, Zimmermann M, Reinhardt D, Hermann J, Berthold F, Henze G, Jurgens H, Kabisch H, Havers W, Reiter A, Kluba U, Niggli F, Gadner H. Improved treatment results in highrisk pediatric acute myeloid leukemia patients after intensification with high-dose cytarabine and mitoxantrone: results of Study Acute Myeloid Leukemia-Berlin-Frankfurt-Munster 93. J Clin Oncol 2001;19:2705-13.

[11] Pawson R, Potter MN, Theocharous P, Lawler M, Garg M, Yin JA, Rezvani K, Craddock C, Rassam S, Prentice HG. Treatment of relapse after allogeneic bone marrow transplantation with reduced intensity conditioning (FLAG \pm Ida) and second allogeneic stem cell transplant. Br J Haematol 2001;115:622-9.

[12] Mitelman F, editor. An international system for human cytogenetic nomenclature. Basel: S. Karger, 1995.

[13] Panarello C, Rosanda C, Morerio C, Russo I, Dallorso S, Gambini C, Ricco AS, Storlazzi T, Archidiacono N, Rocchi M. Lipoblastoma: a case with $\mathrm{t}(7 ; 8)(\mathrm{q} 31 ; \mathrm{q} 13)$. Cancer Genet Cytogenet 1998;102:12-4.

[14] Ishii E, Eguchi M, Matsuzaki A, Zaizen Y, Yoshidomi S, Kimura N, Takeshita M, Tashiro S, Kamada N, Suita S, Ueda K, Miyazaki S. Granulocytic sarcoma in infant with $M L L$ rearrangement preceding acute monoblastic leukemia with $\mathrm{t}(10 ; 11)(\mathrm{p} 11 ; \mathrm{q} 23)$. Leukemia 1995; 9:1970-4.

[15] DiMartino JF, Ayton PM, Chen EH, Naftzger CC, Young BD, Cleary ML. The $A F 10$ leucine zipper is required for leukemic transformation of myeloid progenitors by MLL-AF10. Blood 2002;99:3780-5.

[16] Roll P, Zattara-Cannoni H, Bustos-Bernard MC, Curtillet C, Michel G, Vagner-Capodano AM. Molecular and fluorescence in situ hybridization analysis of a 10;11 rearrangement in a case of infant acute monocytic leukemia. Cancer Genet Cytogenet 2002;135:187-91.

[17] Klaus M, Schnittger S, Haferlach T, Dreyling M, Hiddemann W, Schoch C. Cytogenetics, fluorescence in situ hybridization, and reverse transcriptase polymerase chain reaction are necessary to clarify the various mechanisms leading to an $M L L-A F 10$ fusion in acute myeloid leukemia with 10;11 rearrangement. Cancer Genet Cytogenet 2003; $144: 36-43$. 